\title{
Progressive retinal atrophy in the Abyssinian cat: studies of the DC-recorded electroretinogram and the standing potential of the eye
}

\author{
K L NARFSTRÖM, S E NILSSON, AND B E ANDERSSON \\ From the Department of Ophthalmology, University of Linköping, S-581 85 Linköping, Sweden
}

SUMmARY DC-recorded electroretinography (ERG) and direct recordings of the standing potential (SP) were performed on a group of normal cats and Abyssinian cats affected by a hereditary retinal degenerative disease with similarities to human retinitis pigmentosa. A significant reduction of a- and b-wave amplitudes was found at an early stage of disease at a time when there were no major alterations in the c-wave and SP. At later stages both the c-wave and the SP oscillations were significantly reduced or absent. These findings indicate a primary photoreceptor disorder. Threshold studies for the scotopic b-wave showed a loss of retinal sensitivity early in the disease at a time when $30 \mathrm{~Hz}$ flicker responses were normal, which could indicate an earlier involvement of the rods than of the cones. There were no major alterations in the timing of the ERG in the affected animals tested.

Progressive retinal atrophy (PRA), a slowly progressive retinal degenerative disease, has been described in Abyssinian cats. ${ }^{1-3}$ In a study of 17 parental matings the disease was found to be transmitted by a simple recessive gene. ${ }^{4}$ In affected cats a bilateral retinopathy is usually ophthalmoscopically evident between $1 \frac{1}{2}$ and two years and progresses to cause blindness in another two to three years. In the early stages of the disease the photoreceptor layer in the midperipheral and peripheral region is more severely affected than in the posterior pole. The disease progresses and the retinal degeneration becomes generalised. It was suggested that PRA in the Abyssinian cat is a heritable photoreceptor degenerative disorder with a fairly slow rate of progression. $^{3}$

The present studies were performed to characterise the disease further, to correlate the electrophysiological changes with the disease stage as seen by ophthalmoscopy, and to study the development and progression of electrophysiological abnormalities during the course of the disease. A DC-recorded electroretinography (DC-ERG) technique was used which allows stable and reproducible recordings of the $\mathrm{a}-, \mathrm{b}-$, and $\mathrm{c}$-waves as well as direct recordings of

Correspondence to Dr K L Narfström. the standing potential (SP) of the eye in a clinical situation.

\section{Materials and methods}

Electrophysiological studies were performed in six normal control and seven affected Abyssinian cats (Table 1). Both affected and control cats were household animals and not maintained under standard laboratory animal conditions.

The cats were premedicated with atropine and zylazine (Rompun). General anaesthesia was induced with pentobarbital and maintained with the same substance diluted in Ringer's solution delivered through an infusion pump (infusion dose and rate: $3.5 \mathrm{mg} / \mathrm{kg} / \mathrm{h}$ ). The cats were intubated and their pulse, respiratory rate, and body temperature monitored continuously during the three hours of the procedure. The pupils were dilated with $10 \%$ phenylephrine and $1 \%$ atropine. Dark adaptation time was 45 minutes. DC recordings of the dark adapted ERG and the SP of the eye were studied. The technique has been described previously in more detail. ${ }^{56}$ In summary, a suction contact lens was applied to each eye and a reference chamber placed nasal to the occiput, all of which were connected by saline-agar bridges to matched calomel half-cells, 618 
low-drift DC amplifiers $(300 \mathrm{~Hz}$ cut-off, $12 \mathrm{~dB} /$ octave), a desk top computer system (Hewlett Packard 9826, 9895A, 7225B, 9876A, and 6940B, USA), and analogue tape recorder and a chart recorder. The results of the ERG studies were plotted and stored on floppy discs for later analysis. The contact lenses, which were centred on the visual axis, had a slightly opaque surface to assure an even illumination of the retina. The stimulus light was produced by a $150 \mathrm{~W}$ xenon lamp and was guided to the eyes by quartz fibre optics ending $5 \mathrm{~cm}$ in front of each lens.

The a-, b-, and c-waves of the ERG were recorded in response to 1- second white light stimuli. Light intensities were varied in $0.5 \log$ unit steps by using neutral density filters (Balzer) from slightly below the normal b-wave threshold, set at $25 \mu \mathrm{V}$, to about $7 \log$ relative units above the normal $b$-wave threshold. Stimulus intervals were $60-180$ seconds depending on light intensity. Threshold determinations for the b-wave were followed by amplitude and implicit time studies for the a-, b-, and c-waves over the intensity range. The amplitude of the b-wave was measured from the trough of the a-wave, whereas that of the aand c-waves was measured from the isoelectric line. Implicit time was measured from stimulus onset to the peak of the individual responses. Flicker studies at $30 \mathrm{~Hz}$ were performed with a high intensity of white light (Clinical photic stimulator, Specialized

Table 1 Animals included in the study

\begin{tabular}{|c|c|c|c|c|}
\hline Catno & Breed & Sex & Age & Fundus/stage \\
\hline \multicolumn{5}{|l|}{ Control } \\
\hline N1 & Domestic short-haired & $\mathbf{F}$ & $1 \mathrm{yr}$ & Normal \\
\hline N2 & Abyssinian cross & $\mathbf{M}$ & $2 \mathrm{yr}$ & , \\
\hline N3 & Abyssinian cross & $\mathbf{F}$ & $2 \mathrm{yr}$ & " \\
\hline N4 & Abyssinian (Somali) & $\mathbf{F}$ & $6 \mathrm{mo}$ & , \\
\hline N5 & Abyssinian & $\mathbf{F}$ & $4 \mathrm{yr}$ & $"$ \\
\hline N6 & Abyssinian & $\mathbf{M}$ & $5 \mathrm{yr}$ & $"$ \\
\hline \multicolumn{5}{|l|}{ Affected } \\
\hline A1 & Abyssinian & $\mathbf{M}$ & $6 \mathrm{mo}$ & Stage 1 \\
\hline A2 & $\eta$ & $\mathbf{F}$ & $6 \mathrm{mo}$ & Stage 1 \\
\hline A3 & 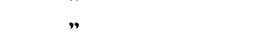 & $\mathbf{F}$ & $11 / 2 \mathrm{yr}$ & Stage 2 \\
\hline A4 & " & $\mathbf{F}$ & $3 \mathrm{yr}$ & Stage 2 \\
\hline A5 & $"$ & $\mathbf{F}$ & $3 \mathrm{yr}$ & Stage 3 \\
\hline A6 & " & $\mathbf{F}$ & $4 \mathrm{yr}$ & Stage 4 \\
\hline A7 & ", & $\mathbf{F}$ & $5 \mathrm{yr}$ & Stage 3 \\
\hline
\end{tabular}

*Stage of disease:

Stage 1 (stage of suspected disease): slight discoloration mainly near the disc.

Stage 2 (stage of early disease): discoloration and slight vessel attenuation most apparent in peripheral and/or midperipheral tapetal fundus.

Stage 3 (stage of moderately advanced disease): distinct colour change in entire tapetal fundus and marked vessel attenuation.

Stage 4 (advanced stage of disease): generalised hyperreflectivity of tapetal fundus, decoloration and sometimes hyperpigmentation of non-tapetal fundus, vessels extremely attenuated or invisible.
Laboratory Equipment, UK, setting 4), clearly within the photopic range. Following dark adaptation the SP was recorded after turning on a continuous white light stimulus (5.67 log relative units above b-wave threshold) for 30 minutes. The maximum amplitude of the light rise was measured from the isoelectric line, and the implicit time from stimulus onset to the peak of the light rise.

DC-ERG recordings were performed on six of the control cats (N1-N6) and flicker and SP recordings on five (N1, N2, N4-N6). Electroretinographic flicker, and SP studies were carried out on all affected
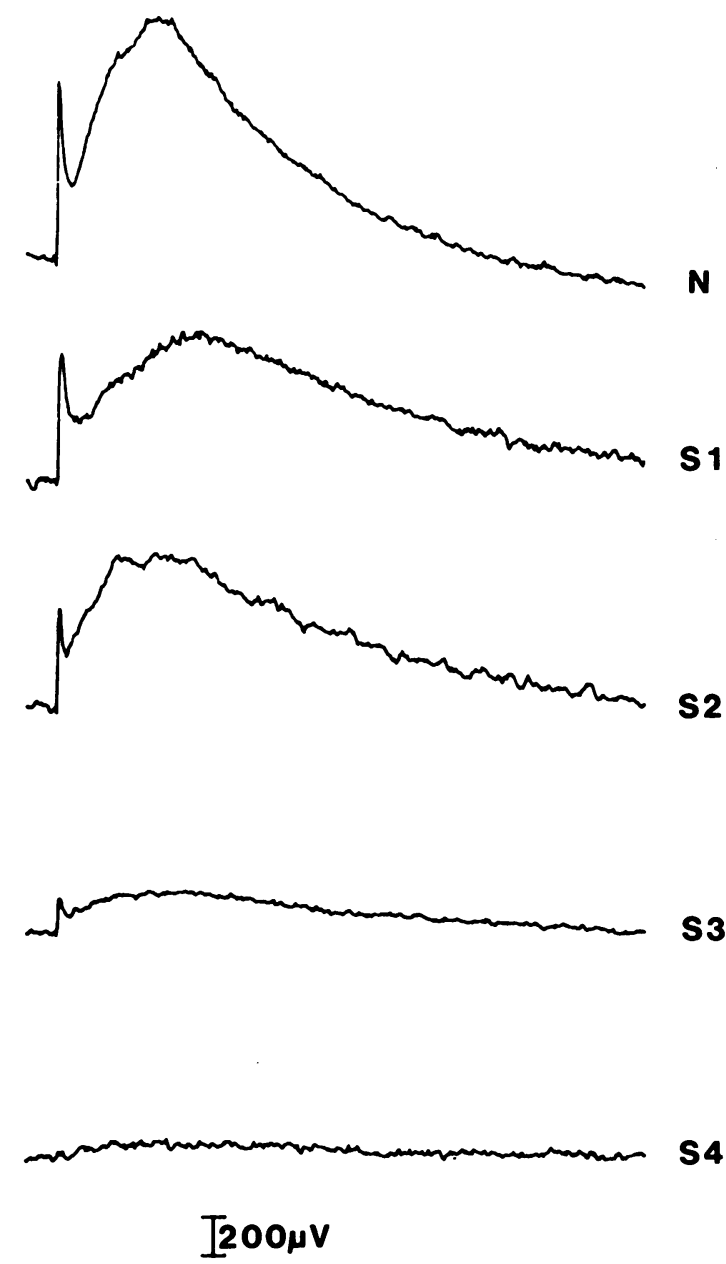

Fig. 1 Dark adapted electroretinogram of a normal Abyssinian (N) and of four affected cats at stages $1-4$ (S1-S4). One second white light stimulus $(5 \cdot 17$ log relative units above b-wave threshold) indicated below recordings. Stimulus conditions identical for all recordings. 
Fig. 2 Log intensity-amplitude (mean and $S E M$ ) relationship for the dark adapted b-wave in normal cats and in affected Abyssians at stages 1-3 (S1-S3). Thresholds (mean and $S E M$ ) are shown parallel to the abscissa. $n=$ Number of cats in each group.

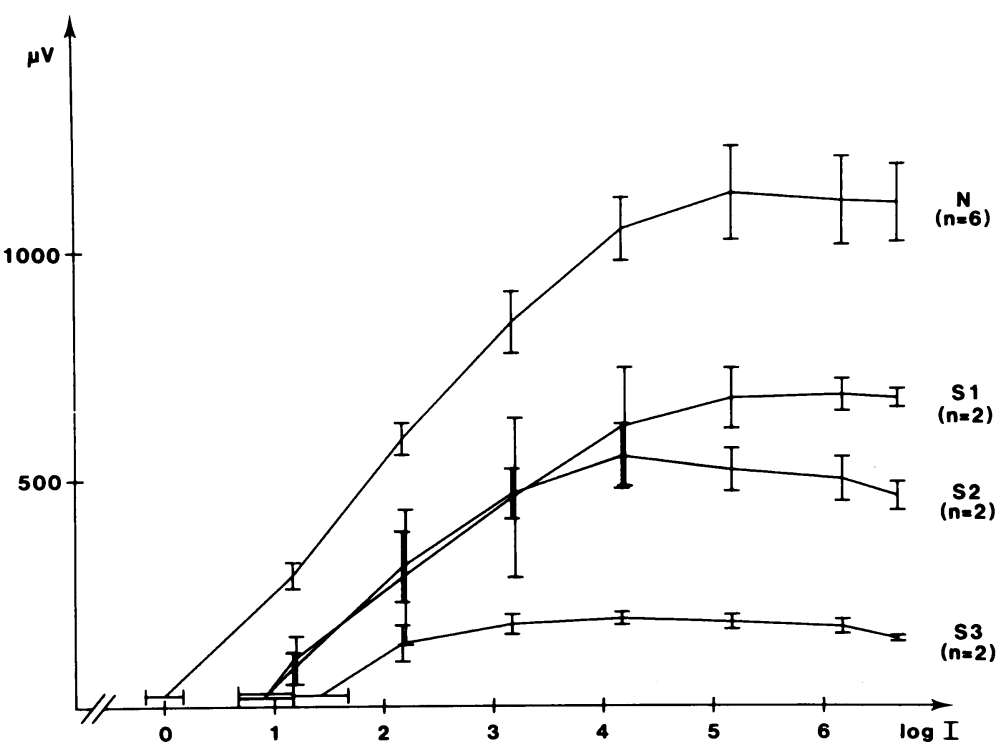

animals (A1-A7). For flicker and SP recordings A1 was used twice-at stages 1 and 2. Data were statistically analysed by the one-tailed Student's $t$ test.

\section{Results}

\section{ERG: THRESHOLD AND AMPLITUDE}

Fig. 1 shows representative recordings from normal and affected cats at different stages of disease.

Fig. 2 illustrates the dark adapted intensityamplitude relationship for the b-wave in normal and affected cats at different stages of the disease. In normal cats the amplitude of the b-wave was linearly related to the logarithm of the stimulus at low light intensities. At higher intensities the relationship became non-linear. The threshold distribution for normal cats was $-0 \cdot 17 \log$ relative units (SEM), with the use of a $25 \mu \mathrm{V}$ b-wave criterion response for threshold. In the affected group (stages 1, 2, and 3) there was an elevation of thresholds which was highly significant $(p<0 \cdot 0005)$. Even at stage 1 the difference was significant $(p<0.02)$ in comparison with the controls. The maximum b-wave amplitude for the intensities used was $1127-103 \mu \mathrm{V}$ (SEM) for the normal group of cats, while for the affected group (stages 1,2 , and 3 ) there was a highly significant reduction $(p<0.0005)$, already noticeable at stage 1 $(p<0.05)$. The reduction in maximum amplitudes corresponded well with the stage of disease: $40 \%$ at stage $1,51 \%$ at stage 2 , and $83 \%$ at stage 3 . In one cat with advanced PRA (stage 4) there was no record- able ERG (not included in the calculations or in the figure).

The dark adapted intensity-response relationship for the a-wave is illustrated in Fig. 3. In normal cats there was a linear increase in amplitudes at higher light intensities, and the maximum amplitude for the intensities used was $283 \pm 39 \mu \mathrm{V}$ (SEM). In the affected group (stages 1,2, and 3) the maximum amplitudes were significantly lower $(\mathrm{p}<0 \cdot 05)$. Again, there was a certain relationship between the maximum amplitudes found and the stage of disease. At stages 1,2 , and 3 the average reduction was $47 \%$, $55 \%$, and $87 \%$, respectively.

Fig. 4 demonstrates the dark adapted intensityreponse relationship for the c-wave in normal and affected cats. The maximum c-wave amplitude for normal cats was $1020 \pm 122 \mu \mathrm{V}$ (SEM). At stages 1 and 2 the reduction in maximum amplitudes was not significant ( $\mathrm{p}<0.25$ for stage 2$)$; at stage 2 the c-wave amplitude was about $21 \%$ of normal. At stage 3, however, there was a significant difference in maximum amplitudes between affected and control animals $(\mathrm{p}<0 \cdot 01)$.

\section{ERG: TIME COURSE}

In both controls and affected cats there was a reduction in implicit time for the a- and b-waves, while for the c-wave there was an increase in implicit time, as higher light intensities were used. There was no major difference in b-wave implicit time between affected cats and controls during scotopic conditions with dim light stimuli. Only at one high light intensity 


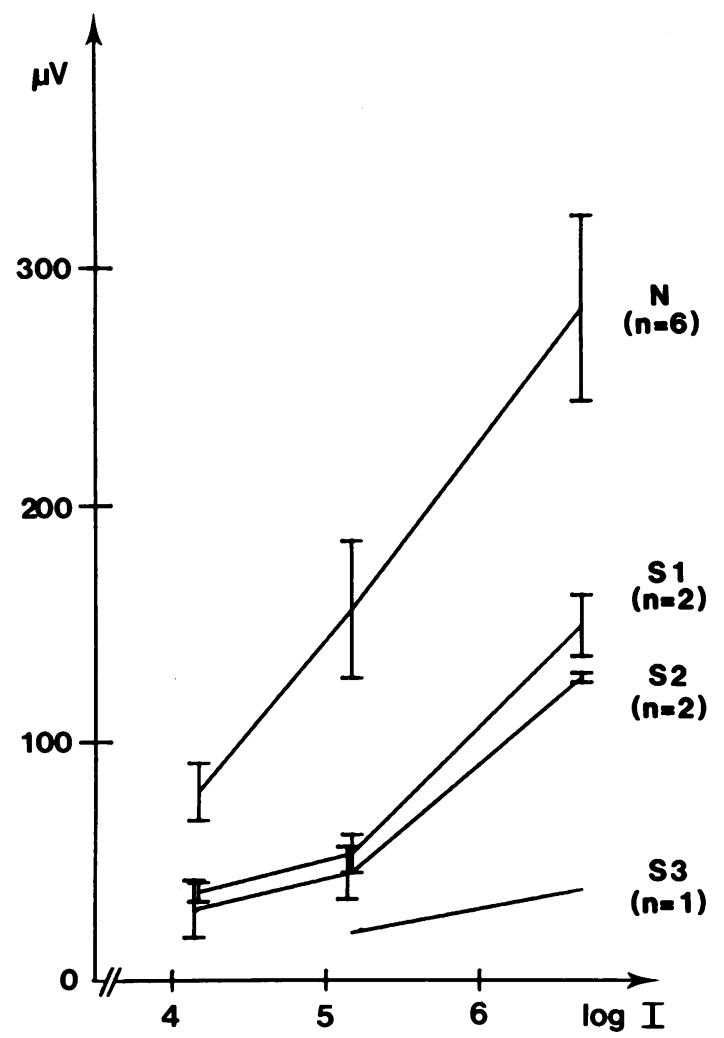

Fig. 3 Log intensity-amplitude (mean and SEM) relationship for the dark adapted a-wave in normal cats and affected Abyssinians at stages 1-3 (S1-S3). n=Number of cats in each group.

(5.17 log relative units above b-wave threshold) was a significant difference in $b$-wave implicit time found $(\mathrm{p}<0.01)$. For the a- and $\mathrm{c}$-waves the implicit times were similar in affected cats and controls at all light intensities used in the study.

\section{ERG: FLICKER}

In control cats the mean b-wave amplitude for $30 \mathrm{~Hz}$ flicker was $16 \pm 1.0 \mu \mathrm{V}$ (SEM), while the mean implicit time was $29 \pm 1 \cdot 0 \mathrm{~ms}$ (SEM). In affected animals the five recordings from stages 1 and 2 showed a mean amplitude of $14 \pm 26 \mu \mathrm{V}$ (SEM) and a mean implicit time of $30.4 \pm 0.7 \mathrm{~ms}$ (SEM). These figures were not significantly different from those of the normal cats. There was no recordable flicker responses in the other affected animals.

\section{SP}

Fig. 5 demonstrates representative SP recordings from a control cat and two affected animals at stages 1 and 2 . In normal cats a light rise with a distinct peak was seen with a mean amplitude of $2.5 \pm 1.5 \mathrm{mV}$ (SEM) and a mean implicit time of $5.3 \pm 1.4 \mathrm{~min}$. (SEM). Further, a less prominent second oscillation was seen in some of the normal cats at about 26 minutes. For affected cats the five recordings at stages 1 and 2 showed a mean amplitude of $2.7 \pm 0.5 \mathrm{mV}$ (SEM) and a mean implicit time of $6.5 \pm 0.5 \mathrm{~min}$ (SEM). These figures do not differ significantly from those of the normal cats. At stages 3 and 4 no light rise was seen.

\section{Discussion}

The successive reduction mainly of a- and b-wave amplitudes indicates that the retinal degeneration found in this strain of Abyssinian cats is primarily a photoreceptor disorder. Threshold studies for the dark adapted b-wave showed that there was a loss in retinal sensitivity early in the disease process, indicating an early involvement of the rod system. The cone system seemed, however, to be spared in early cases, as seen by a normal $30 \mathrm{~Hz}$ flicker response. For the c-wave and SP there were no major alterations at stages 1 and 2, which showed a good function of the pigment epithelium in early cases. With progression of disease, when there was a substantial reduction in a- and b-wave amplitudes, there was also a significant reduction of the c-wave amplitude and an abnormal SP response, which possibly might indicate an involvement of the pigment epithelium as well. However, since both the c-wave and the SP reflect an

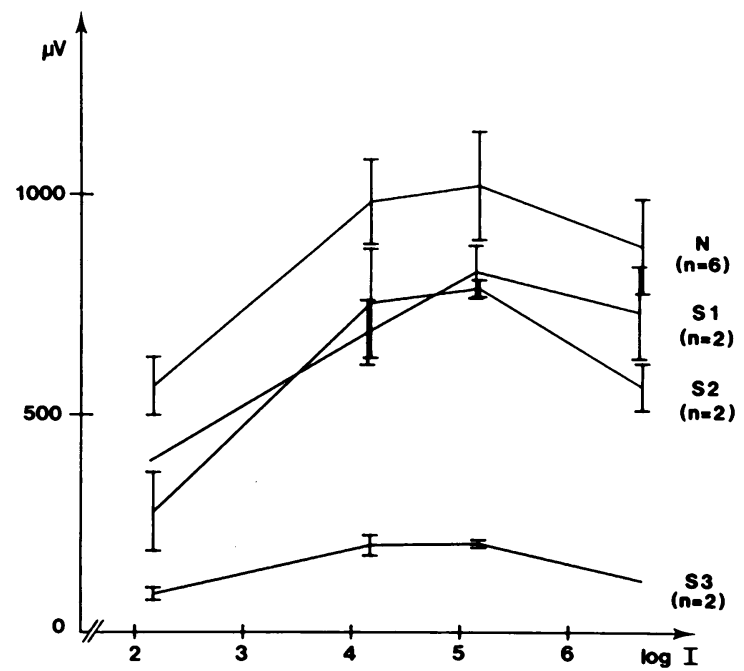

Fig. 4 Log intensity-amplitude (mean and SEM) relationship for the dark adapted c-wave in normal cats and affected Abyssinians at stages 1-3 (S1-S3). n=Number of cats in each group. At $2 \cdot 17 \log I$ units for $S 1$ and at $6 \cdot 67 \log I$ units for $S 3$ the results of one cat were recorded. 

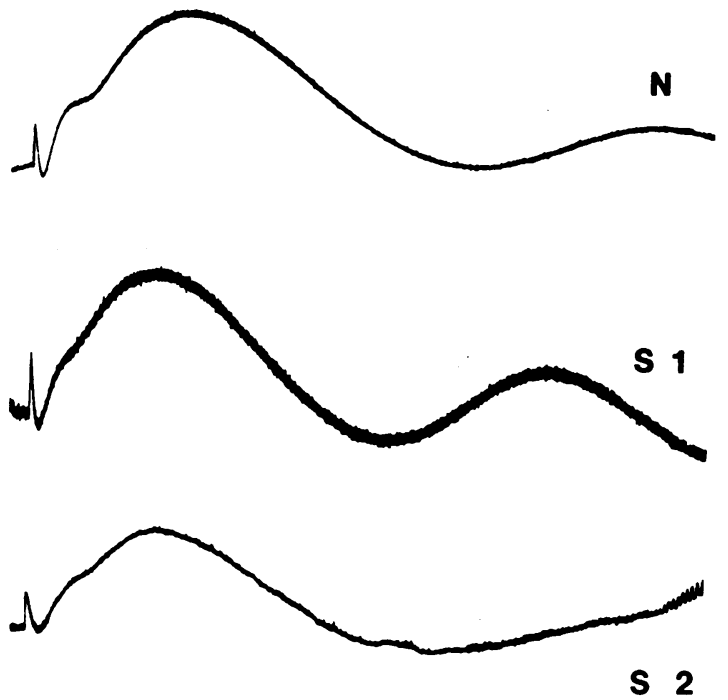

$\uparrow$

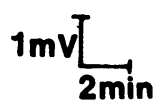

Fig. 5 Representative SP recordings of a normal Abyssinian cat $(N)$ and of affected animals at stages 1 and 2 (S1-S2). The recordings were performed, following dark adaptation, after turning on a continuous white light stimulus (5.67 log relative units above $b$-wave threshold) for 30 minutes.

interaction between neuroretina and pigment epithelium, ${ }^{7-9}$ these alterations could be mainly secondary to the photoreceptor dysfunction.

Results of histopathological studies presented previously, ${ }^{3}$ support the electrophysiological findings in this study. Light microscopy at the early stage of disease showed alterations in the photoreceptor layer, while other parts of the retina, including the pigment epithelium, appeared normal. The midperipheral and/or peripheral retina were most severely affected; they showed a reduced outer nuclear layer and disorganisation and shortening of outer and inner segments, while there were seemingly normal photoreceptors in the central retina. With progression of disease there was an increased loss of visual cell nuclei, and outer and inner segments were degenerating or lacking in most parts of the retina. The end stage showed a generalised loss of photoreceptors with the external limiting membrane in close contact with the pigment epithelium. Abnormalities were not found in the pigment epithelium, as seen by light microscopy, at any stage of the disease process. Electron microscopy (to be published) of early cases confirmed that rods were affected earlier than cones but that there was an involvement of both rods and cones at later stages.
A fairly good correlation was found between the stage of disease, as seen by ophthalmoscopy, and functional integrity of the retina, shown by the intensity-amplitude relationship for the dark adapted ERG. The stages of the disease corresponded more to the rod system than to the cones, which was found by comparing scotopic b-wave and cone flicker responses.

The time course of the ERG did not show any major abnormalities. Dim light stimuli during scotopic conditions and $30 \mathrm{~Hz}$ flicker stimuli showed a normal implicit time for rods and cones, respectively, in affected animals. A significant delay in b-wave implicit time was found at one of the high light intensities only. Previous studies have shown a delay in rod and cone b-wave implicit time in conjunction with generalised photoreceptor disorders. ${ }^{10}$ " More recently, however, normal rod and cone implicit times were found in miniature poodles affected by a generalised retinal degenerative disease. ${ }^{12}$

The retinal degeneration found in this strain of Abyssinian cats shows many similarities to classical retinitis pigmentosa in man. In the latter disease, first the midperipheral and then the peripheral retina is most frequently affected prior to that of the posterior pole, with a progressive loss of night vision. Rods are more severely affected than cones. ${ }^{13}$ Low-voltage ERGs or non-recordable ERGs are frequently found in RP patients, though in early cases ERGs with only slight alterations have been seen occasionally. ${ }^{14}$ is $\mathrm{It}$ is conceivable that this strain of Abyssinian cats may be a new and important animal model for the study of this devastating retinal disease in man.

This work was supported by grants from the Swedish Medical Research Council (Project No. 12X-734) and from the Swedish University of Agricultural Sciences. The work was made possible through the interest and co-operation of Abyssinian cat breeders in Sweden.

\section{References}

1 Narfström K. Progressiv retinal atrofi hos abessinierkatt. Svensk Veterinärtidning 1981; 33: 147-50.

2 Narfström K, Nilsson SE. Progressive retinal atrophy in the Abyssinian cat: an update. Vet Rec 1983; 112: 525-6.

3 Narfström K. Progressive retinal atrophy in the Abyssinian cat. Clinical characteristics. Invest Ophthalmol Visual Sci 1985; 26: 193-200.

4 Narfström K. Hereditary progressive retinal atrophy in the Abyssinian cat. J Hered 1983; 74: 273-6.

5 Nilsson SE, Skoog KO. Covariation of the simultaneously recorded c-wave and standing potential of the human eye. Acta Ophthalmol (Kbh) 1975; 53: 721-30.

6 Skoog KO. The directly recorded standing potential of the human eye. Acta Ophthalmol (Kbh) 1975; 53: 120-32.

7 Steinberg RH, Schmidt R, Brown KT. Intracellular responses to light from cat pigment epithelium: origin of the electroretinogram c-wave. Nature 1970; 227: 728-30. 
8 Oakley B II, Green DG. Correlation of light-induced changes in retinal extracellular potassium concentration with c-wave of the electroretinogram. J Neurophysiol 1976; 39: 117-33.

9 Steinberg RH, Linsenmeier RA, Griff ER. Three light evoked responses of the retinal pigment epithelium. Vision Res 1983; 23: 1315-23.

10 Berson EL, Gouras P, Gunkel RD. Rod responses in retinitis pigmentosa dominantly inherited. Arch Ophthalmol 1968; 80: 55-67.

11 Berson EL, Gouras P, Hoff M. Temporal aspects of the electroretinogram. Arch Ophthalmol 1969; 81: 207-14.
12 Pawlyk B, Sandberg HA, Berson EL. Temporal aspects of the electroretinogram of miniature poodles with progressive retinal atrophy. Invest Ophthalmol Visual Sci 1984; 25 (suppl): 196.

13 Krill A, Archer DB, ed. Krill's hereditary retinal and choroidal diseases. London: Harper and Row, 2: 1977.

14 Berson EL. Electrical phenomena in the retina. In: Moses RA, ed. Adler's physiology of the eyé. Saint Louis: Mosby, 1975.

15 Marmor MF. The electroretinogram in retinitis pigmentosa. Arch Ophthalmol 1974; 97: 1300-4. 